\title{
Globalization effects on the reports of non-endemic parasitosis in Italy
}

\author{
Giorgia Bracaglia, ${ }^{1}$ Livia Mancinelli, ${ }^{2}$ Stefania Ranno, ${ }^{2}$ Federica Del Chierico, ${ }^{3}$ Hyppolite K. Tchidjou, ${ }^{4}$ \\ Ottavia Porzio, ${ }^{1}$ Andrea Onetti Muda, ${ }^{5}$ Lorenza Putignani ${ }^{2,3}$ \\ ${ }^{1}$ Laboratory Medicine, ${ }^{2}$ Unit of Microbiology, Parasitology and Virology, ${ }^{3}$ Human Microbiome Unit, ${ }^{4}$ Unit of Immunology \\ and Infectious Diseases, ${ }^{5}$ Department of Laboratories, Bambino Gesù Children's Hospital, IRCCS, Rome, Italy
}

\section{Summary}

Protozoa and helminths are responsible for several intestinal parasite infections (IPIs). Generally, helminth infections are very unsafe but scarcely reported in high-income countries, while protozoa and helminth co-infections are usually reported in children living in inadequate hygienic-sanitary conditions and in rural areas. However, the impact of growing globalization, intense travelling, international adoptions and high levels of immigrants and refugees has significantly incremented the incidence of orofecal parasitosis in non-endemic areas. Although most IPs clear without treatment when population, even children, emigrate from endemic to different geographical areas, some IPIs such as strongyloidiasis may persist for decades as subclinical infections or as low-grade disease with nonspecific clinical manifestations, unless to reappear under impairment conditions. Herein we report an unusual case of Giardia lamblia and Trichuris spp. chronic asymptomatic co-infection in a healthy adopted Romanian child, living in a

Correspondence: Lorenza Putignani, Unit of Microbiology, Parasitology and Virology, Bambino Gesù Children's Hospital, IRCCS, Piazza Sant'Onofrio 4, 00165, Rome, Italy.

Tel.: +39.06.68592598/2980.

E-mail: lorenza.putignani@opbg.net

Key words: International adoptions; asymptomatic and misidentified parasitosis; intestinal parasite infections, IPIs; Giardia lamblia; Trichuris spp.; Strongyloides stercoralis; IPIs screening guidelines.

Contributions: LM and SR contributed equally.

Conflict of interest: the authors declare no potential conflict of interest.

Funding: This work was supported by the Ministry of Health, Ricerca Corrente (RC201302P002991) Bambino Gesù Children's Hospital, IRCCS, and by Dicofarm Grant to LP.

Received for publication: 11 November 2018.

Accepted for publication: 3 December 2018.

CCopyright G. Bracaglia et al., 2018

Licensee PAGEPress, Italy

Microbiologia Medica 2018; 33:7932

doi:10.4081/mm.2018.7932

This article is distributed under the terms of the Creative Commons Attribution Noncommercial License (by-nc 4.0) which permits any noncommercial use, distribution, and reproduction in any medium, provided the original author(s) and source are credited.
Central Italy rural area, and a hidden case of Strongyloides stercoralis in an adopted Burundian child, resident in South Italy, long misdiagnosed as a recurrent undefined dermatitis. Our report suggests the need to review primary care practitioner guidelines and children's hospital procedures for appropriate IPIs screening and follow-up, hence providing new screening and prevention strategies, in agreement with international guidelines.

\section{Introduction}

Protozoa and helmints are responsible for several intestinal parasite infections (IPIs), especially in children (2), even occurring after ingestion of only few cysts or eggs, related with several factors such as age, patient's immune status and nutritional conditions. Parasites frequently detected in stools are protozoa (e.g., Giardia lamblia, Entamoeba histolytica, Cryptosporidium spp.) (11) and soil-transmitted helminths (STH) (e.g., Ascaris, whipworms, and hookworms) (9). Particularly, G. lamblia is a flagellate affecting people worldwide, especially children living in rural areas with inadequate hygienic conditions or attending school in social contaminated environments (1).

Risk of helminthiasis has been associated to wastewater and excreta contamination in agricultural settings, suggesting water treatments as key factors to reduce infestations (9). Helminths are parasites infecting humans especially in developing countries which can cause several clinical troubles such as anemia, growth retardation and increased susceptibility to other infections (6).

In Italy, a study including 5323 patients has found that G. lamblia is the most common protozoan in both Italian and non-Italian patients, while worms, particularly Hymenolepis nana, Strongyloides stercoralis and Trichuris trichiura, are found only in $0.9 \%$ of cases, especially in foreign children (16).

Herein we report a G.lamblia and Trichuris spp. co-infection and a $S$. stercoralis misdiagnosed infection, two cases found in adopted children living in Italy and coming from endemic regions for IPIs. The aim is to discuss new alert algorithms for IPIs in our country by implementing pediatric screening programs for adopted and migrant children from endemic areas, within the context of specialized reference Centers for children parasitosis control.

\section{Ethics approval and consent to participate}

Written informed consent was obtained from the patient's parents for inclusion in the study and for publication of this manuscript. The study was conducted in accordance with the Declaration of Helsinki, and the protocol was approved by the Bambino Gesù Children's Hospital Ethics Committees. 


\section{Case Report \#1}

We present an 8-years-old boy, adopted from Hungary by an Italian family, living near Simbruini Mounts (Lazio-Italy) since May 2012. Except for recurrent respiratory tract diseases, his medical history was negative for viral, bacterial and parasitic infections and all recommended vaccines were accomplished. He was brought to the attention of pediatricians of Bambino Gesù Children's Hospital (OPBG) of Rome, for routine screening evaluations, including a complete physical examination, which resulted normal with respect to age. Laboratory tests for HIV, HBV, $\mathrm{HCV}$, tuberculosis and immunological status resulted negative. However, parasitological analyses resulted positive for G. lamblia and Trichuris spp. Therefore, the child was treated with mebendazole for 5 days and probiotics for 14 days. Three stool samples, collected at alternate days and fixed in $10 \%$ formalin, were analyzed, after concentration, for clinical microscopy workflow under a 100× light microscope with micrometric eyepiece, in accordance with Ritchie and stained with Lugol $(14,20)$. Ten slides for sample were set-up for microscopy. A first slide revealed one G. lamblia cyst of $12 \times 10 \mu \mathrm{m}$ size (Figure 1A) and a brown lemon-shaped egg of Trichuris spp. of $52 \times 23 \mu \mathrm{m}$ size (Figure 1B). In the other slides a total of 15 cysts of G. lamblia, 6 eggs and 2 larval forms of Trichuris spp. were observed (Figure 1C-E). Each fecal sample was also processed through rapid immunocromatographic test (ICT), Rida quick immunochromatographic dipstick tests Cryptosporidium/Giardia/Entamoeba Combi (R-Biopharm, Darmstadt, Germany), which resulted positive for Giardia antigen.

All samples were processed, according to routine barcode-based automatic DNA extraction, to perform molecular assays on Giardia isolates (QIAsymphony SP/AS Instruments, Qiagen, Hilden, Germany). A G. lamblia molecular typing was performed by multilocus PCR analysis based on: i) a nested-PCR procedure, amplifying a DNA region of $130 \mathrm{bp}$ (18rRNA small subunit, SSU) (Figure 2A) (12) (i.e., genotyping) ; ii) a semi-nested PCR procedure, amplifying a $432 \mathrm{bp}$ region of the glutamate dehydrogenase gene $(g d h)$ (Figure 2B) (13) (i.e., sub-genotyping). The PCR products were purified using centrifugal filter Amicon Ultra according to the manufacturer's instructions (Millipore, Carrigtwohill, Ireland) to proceed with amplicon characterization. Sequencing reactions were performed using BigDye terminator v.3.1 Sequencing kit, following the manufacturer's instruction (Applied Biosystems, CA, USA). G. lamblia sequences were corrected by base calling, edited using FinchTV 1.4 software (Geospiza, Inc, Seattle, WA, USA), and queried using BLAST (http://www.ncbi.nlm.nih.gov/BLAST) to infer similarities of forward/reverse consensus sequences to assign species/assemblage/sub-assemblage ranking. Nucleotide gdh sequences were deposited into GenBank (BankIts: KF317538; KF317539; KF317540). In order to underline phylogenetic relationships, software MEGA Version 5.05 was used and the best tree construction model test (model T92, Tamura 3-parameter) was performed for the dataset (Figure 2C). All three G. lamblia isolates resulted sub-genotyped as BIII assembly group. On the other end, under routine laboratory procedures, Trichuris DNA extraction failed, as ascertained by STH-based amplification, possibly because the automatic DNA extraction was unsuitable for Trichuris spp. (7). Therefore, only clinical microscopy was exploited for STH diagnosis.

After therapy, the patient not returned at control as recommended. However, after one year, during a routine control, physical examination showed normal height-weight development and absence of disease. Parasitological exams showed absence of Giardia infection, but still presence of Trichuris spp. ova. However, consistently with literature data, clinicians decided to reserve treatment only in the case of further presenting symptoms (18).

\section{Case Report \#2}

We also report on a 2-years-old child coming from Burundi, arrived in Italy in April 2012 and hospitalized for hypereosinonophilic syndrome and recurrent dermatitis in Potenza, where he was treated for itchy hives for several months with steroids and antihistamines, without remission of symptoms. All vaccinations were completed. At the arrive to our OPBG Hospital the child showed good conditions, except for skin lesions due to previous skin infection scarring. Hematological parameters revealed hypereosinonophilia (eosinophil count $3.66 \times 103 / \mu \mathrm{L}, 42.1 \%$ reference values, RV), lymphopenia (lymphocyte count $2.48 \times 103 / \mu \mathrm{L}, 28.5 \%$ $\mathrm{RV}$ ) and neutropenia (neutrophils count $2 \times 103 / \mu \mathrm{L}, 23 \% \mathrm{RV}$ ).

Parasitological exams and routinely DNA-extraction procedures were performed by clinical microscopy and molecular biology pipelines. Only one, amongst the multiple analyzed slides,

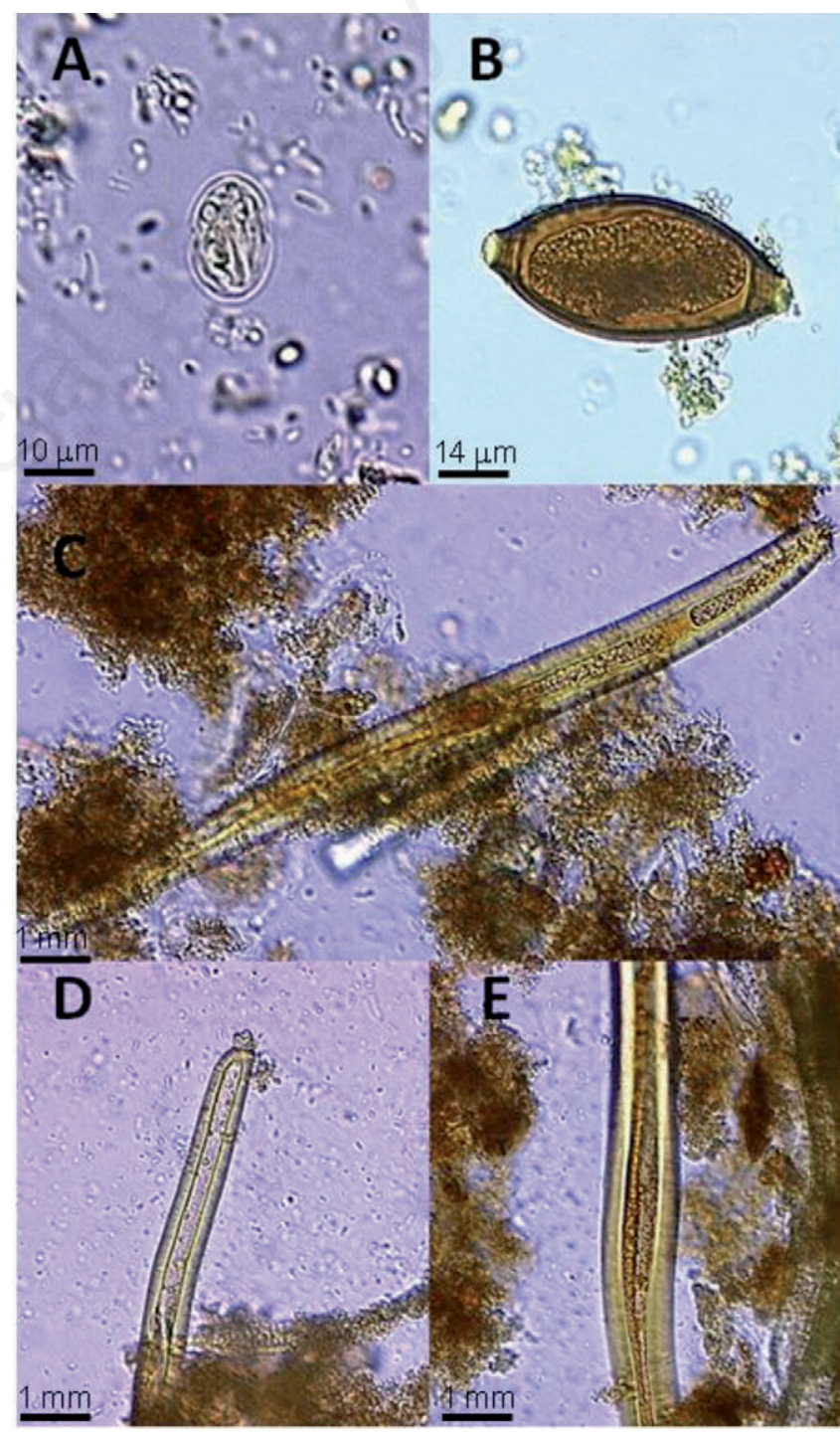

Figure 1. Microscope-based identification of Giardia lamblia and Trichuris trichiura in concentrated Lugol-stained samples. (A) Cyst of G. lamblia; (B) egg of T. trichiura; (C) adult worm of T. trichiura; (D) mouthparts of T. trichiura; (E) intestinal apparatus of T. trichiura. 
showed the presence of a $S$. stercoralis larva (Figure 3A). S. stercoralis PCR was performed using specific STRO 1530 forward and STRO 1630 reverse primers (4), amplifying a 18rRNA SSU fragment of $114 \mathrm{bp}$, which resulted consistent with the size of the internal positive control (IPC) (4) (Figure 3B). All microscopic and molecular data correlated with the presence of body erythematous lesions associated to a cutaneous larva migrans (Figure 3C).

\section{Results and Discussion}

The presented two cases to focus on childhood health state in poor areas and during immigration/adoption flows, in order to realize appropriate disease control and prevention measures for adopted or migrant children. Helminthiasis and protozoan diseases cover a significant infectious disease burden and many are the parasites responsible for IPIs leading to persistent diarrhea and/or major complications. Epidemiological data on IPIs disease diffusion and prevalence in developing countries are numerous, while in high income countries such infections are rarely reported (8).

Microscopical analysis, coupled to DNA-based typing for $G$. lamblia, allowed us to describe a G. lamblia and Trichuris spp. coinfection in an adopted asymptomatic child. This was an unusual co-infection morbidity in Italy, although frequently association between protozoa and helminths have been widely described in literature $(2,16)$. Indeed, in a study from Ancona Hospital, in 20062011, protozoa and helminth co-infections were reported in children, especially in strangers (16) and G. lamblia was detected with Taenia spp. and $S$. stercoralis eggs, but not with other worms. Moreover, T. trichiura has never been reported with amoebae. $G$. lamblia is genetically considered a multi-species complex which
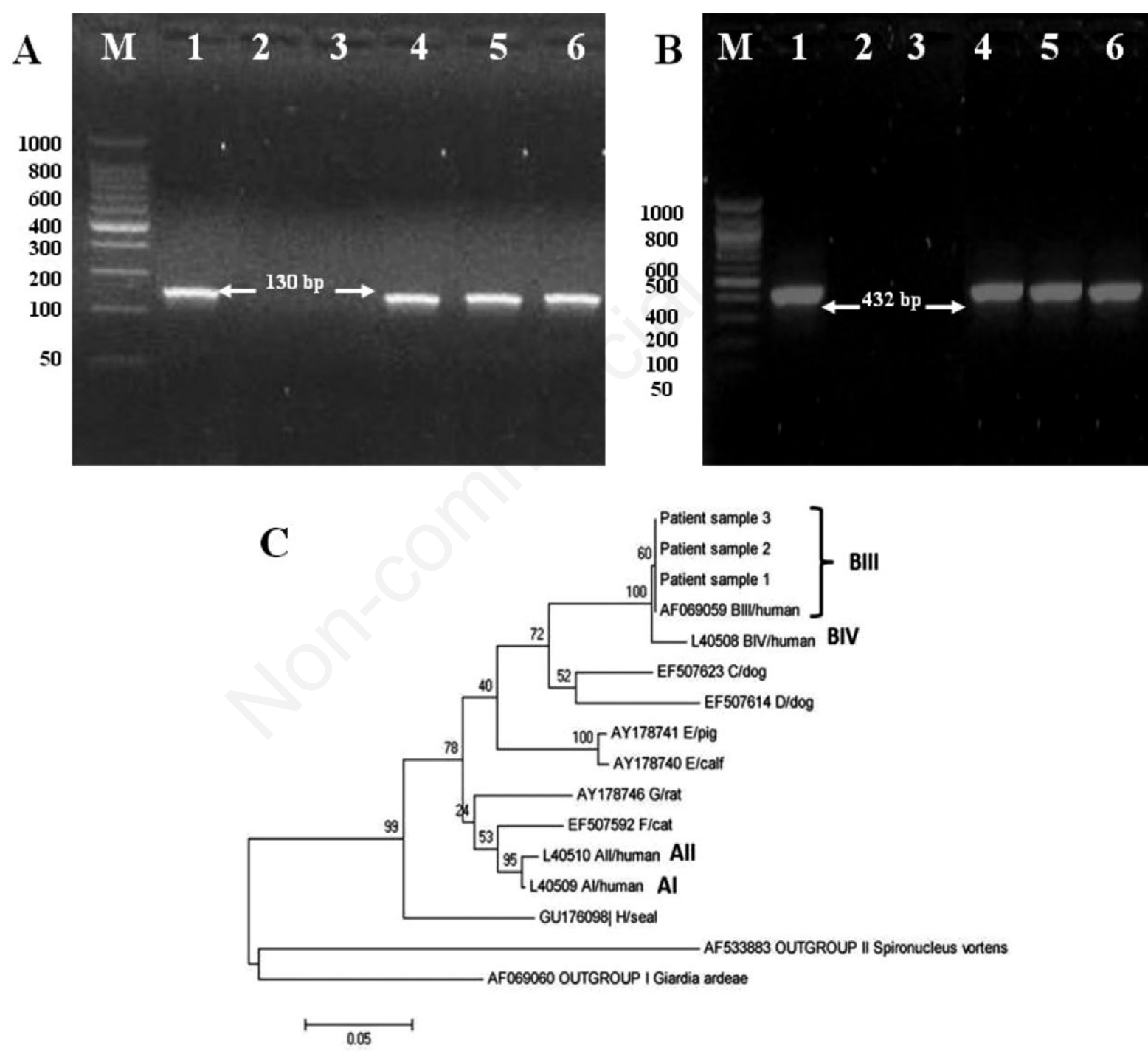

Figure 2. Molecular analysis of Giardia lamblia. (A) Gel representation of the SSU-18rRNA-based PCR for G. lamblia assemblage detection: in order (M) marker, (1) internal positive control, (IPC) (2) first PCR negative control, (3) nested-PCR negative control, (4) patient sample 1, (5) patient sample 2, (6) patient sample 3. (B) Gel representation of the gdh-based PCR for G. lamblia sub-assemblage detection: in order (M), marker, (1) IPC, (2) first PCR negative control, (3) nested-PCR negative control (4) patient sample 1, (5) patient sample 2, (6) patient sample 3. (C) Neighbour-joining phylogenetic tree based on a ClustalW alignment of the gdh sequences from the three $G$. lamblia patient isolates. The percentage of replicate trees in which the associated taxa clustered together in the bootstrap test (1000 replicates) is shown above branches. 
includes a variety of morphologically similar genotypes differing for host specificity (19). Molecular genotyping of isolates, coming from different hosts, has allowed so far, the identification (ID) of seven assemblages (e.g., A-G) (19). The A and B assemblages are considered to be potentially zoonotic, because of their ID in both humans and animals and they include AI, AII, AIII, and BIII and BIV sub-assemblage groups, respectively. The PCR-based analysis for G. lamblia, from our stool samples, confirmed by microscopybased positivity, and performed by exploiting both 18rRNA SSU and $g d h$ gene targets, assigned assemblage B and sub-assemblage BIII. The BIII sub-assemblage ID (17), seems to suggest the hypothesis of a zoonotic transmission, supported by the patient behavior within his socio environmental conditions. Also, infections by Trichuris spp. primarily affect people living in rural areas, particularly people in contact with domestic animals (5); in the herein presented case the possible reservoir of parasite infection might had been the child's dog. In Italy, indeed, a previous study (3) reported the presence of mixed trichuroid infestation in an asymptomatic dog suggesting a zoonotic risk.

In the second case, we reported a $S$. stercolaris infection in a child from Burundi, treated for several months with antihistaminic and corticosteroid therapy, because of a suspected allergy reaction. When the child arrived to hospital, parasitological exams showed the presence of one only larva of $S$. stercolaris in just one slide, regardless several stool collection and multiple slide sets for each microscopic session. Symptoms were solved after specific albendazole treatment with complete eradication of STH parasite at control six months later, with remission of skin lesions due to larva migrans and resolution of haematological parameters.

\section{Conclusions}

These cases show the importance to deeply examine by dedicated parasitological protocols healthy children with history of migration of international adoption, hence avoiding further aggressive treatments for hidden or neglected parasitosis.

Our laboratory is focused on the evaluation of prevalence and incidence of pediatric parasitosis, paying remarkable attention to new IPIs, currently circulating in Italy as effect of globalization processes. In fact, in a previous study we have reviewed the prevalence of parasitic infections in Italy and in high income countries by effect of globalization phenomena (11). In addition, it has been demonstrated in a retrospective study on 504 adopted children that G. lamblia was detected in 87 out of 461 tested children, especially native of Eastern Europe $(15,19)$, as in the case of our patient. When populations emigrate from parts of the world where intestinal parasites are endemic and resettle in countries where they do not exist or are rare, most infections will clear without treatment with-
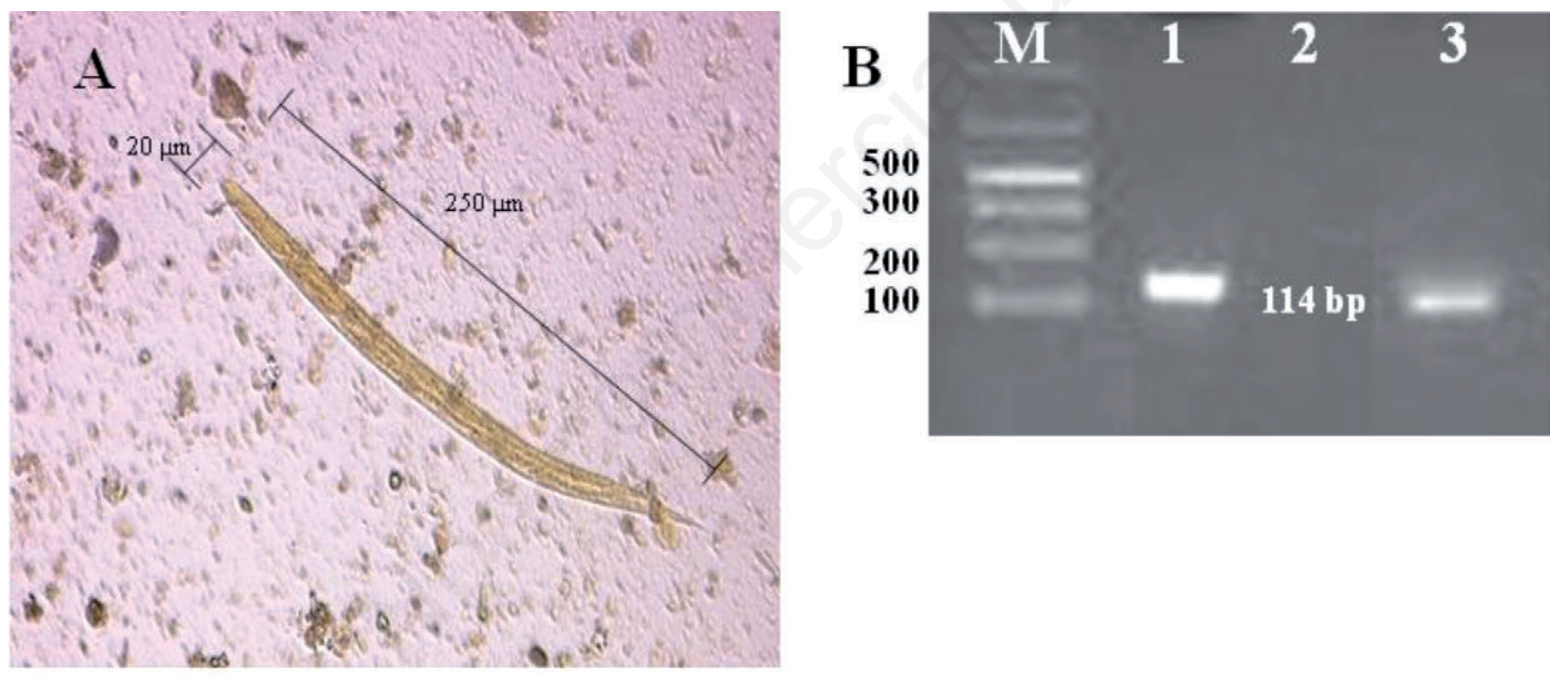

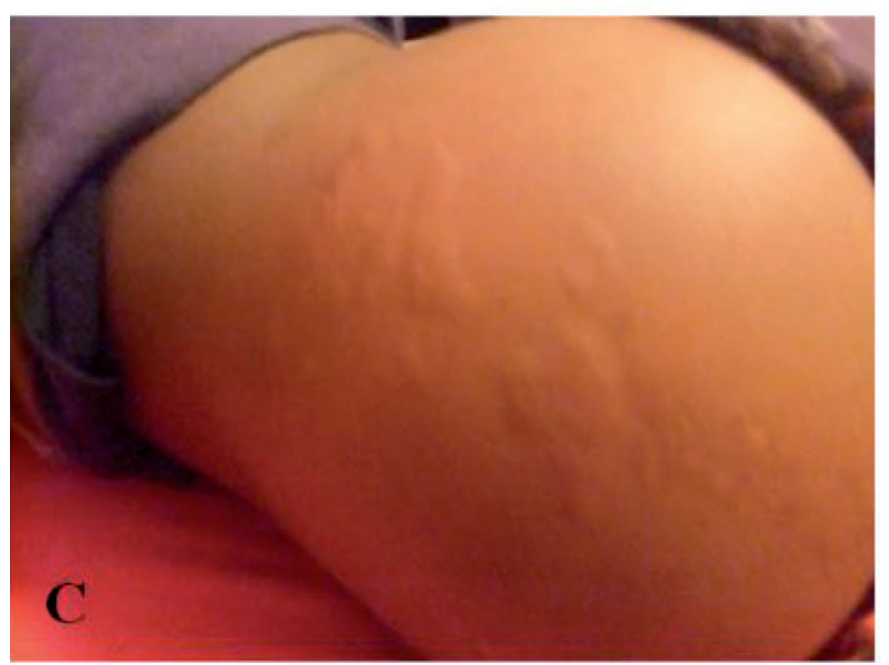

Figure 3. Microscope- and molecular-based identification of Strongyloides stercoralis coupled to clinical inspection of larva migrans detectable at cutaneous level. (A) Filariform (L3) larvae of S. stercoralis in Lugol staining. (B) Electrophoresis gel for $S$. stercoralis detection: (M) marker, (1) Patient sample, (2) Negative Control, (3) Internal positive control (IPC) for S. stercoralis DNA. (C) Cutaneous larva migrans. 
in a few years after immigration $(20,10)$. However, some IPIs agents may persist for decades as subclinical infections or as lowgrade disease with nonspecific symptoms.

To prevent and to control neglected IPIs associated to environmental and/or sociological infestations (i.e., anthroponotic and zoonotic transmission), it is now mandatory to design new advanced diagnostic and clinical prevention strategies when history of migration can actually interfere with the forthcoming child health status. Especially Pediatric Hospitals should put into effect new clinical protocols and diagnostic algorithms able to detect neglected parasitosis, often asymptomatic and misidentified or, even, hidden. Diagnostic workflows need now to combine serology, molecular biology and clinical microscopy handled by well trained laboratory staffs especially when medical history is not available or confused by scarceness of clinical data.

\section{References}

1 Barry MA, Weatherhead JE, Hotez PJ, Woc-Colburn L. Childhood parasitic infections endemic to the United States. Pediatr Clin North Am 2013;60;471-85.

2. Brown J, Cairncross S, Ensink JH. Water, sanitation, hygiene and enteric infections in children. Arch Dis Child 2013 Jun 12.

3. Di Cesare A, Castagna G, Meloni S, et al. Mixed trichuroid infestation in a dog from Italy. Parasit Vectors 2012;5:128.

4. Dorris M, Blaxter $M$. The small subunit ribosomal RNA sequence of Strongyloides stercoralis. Int J Parasitol 2000;30:939-41.

5. Dunn JJ, Columbus ST, Aldeen WE, et al. Trichuris vulpis recovered from a patient with chronic diarrhea and five dogs. J Clin Microbiol 2002;40;2703-4.

6. Hotez PJ, Brooker S, Bethony JM, et al. Hookworm infection. N Engl J Med 2004;351:799-807.

7. Liu GH, Gasser RB, Su A, et al. Clear genetic distinctiveness between human- and pig-derived Trichuris based on analyses of mitochondrial datasets. PLoS Negl Trop Dis 2012;6:e1539.

8. Peruzzi S, Gorrini C, Piccolo G, et al. Prevalence of intestinal parasites in the area of Parma during the year 2005. Acta Biomed 2006;77:147-51.
9. Pham-Duc P, Nguyen-Viet H, Hattendorf J, et al. Ascaris lumbricoides and Trichuris trichiura infections associated with wastewater and human excreta use in agriculture in Vietnam. Parasitol Int 2013;62:172-80.

10. Pottie K, Greenaway C, Feightner J, et al. Coauthors of the Canadian Collaboration for Immigrant and Refugee Health Evidence-based clinical guidelines for immigrants and refugees CMAJ 2011;183:E824-925.

11. Putignani L, Menichella D. Global distribution, public health and clinical impact of the protozoan pathogen Cryptosporidium. Interdiscip Perspect Infect Dis 2010;2010:753512.

12. Read C, Walters J, Robertson ID, Thompson RC. Correlation between genotype of Giardia duodenalis and diarrhea. Int $\mathrm{J}$ Parasitol 2002;32:229-31.

13. Read CM, Monis PT, Thompson RC. Discrimination of all genotypes of Giardia duodenalis at the glutamate dehydrogenase locus using PCR-RFLP. Infect Genet Evol 2004;4:125-30.

14. Ritchie L. An ether sedimentation technique for routine stool examination. Bull US Army Med Dep 1948;8;326.

15. Saiman L, Aronson J, Zhou J, et al. Prevalence of infectious diseases among internationally adopted children. Pediatrics 2001;108:608-12.

16. Silvestri C, Greganti G, Arzeni D, et al. Intestinal parasitosis: data analysis 2006-2011 in a teaching hospital of Ancona, Italy. Infez Med 2013;21:34-9.

17. Souza SL, Gennari SM, Richtzenhain LJ, et al. Molecular identification of Giardia duodenalis isolates from humans, dogs, cats and cattle from the state of São Paulo, Brazil, by sequence analysis of fragments of glutamate dehydrogenase (gdh) coding gene. Vet Parasitol 2007;149:258-64.

18. Tahas SA, Diakou A. Persistent Giardia spp. and Trichuris spp. infection in maras (Dolichotis patagonum) at a zoo in Greece. J Zoo Wildl Med 2013;44:389-94.

19. Thompson RC. Giardiasis as a re-emerging infectious disease and its zoonotic potential. Int J Parasitol 2000;30:1259-67.

20. Vandenberg O, Van Laethem Y, Souayah H, et al. Improvement of routine diagnosis of intestinal parasites with multiple sampling and SAF-fixative in the triple-faeces-test. Acta Gastroenterol Belg 2006;69:361-6. 